\title{
Combination of two Gas Electron Multipliers and a Micromegas as gain elements for a time projection chamber
}

\author{
S. Aiola ${ }^{a}$, R.J. Ehlers ${ }^{\mathrm{a}}$, S. Gu ${ }^{\mathrm{a}}$, J.W. Harris ${ }^{\mathrm{a}}$, R. Majka ${ }^{\mathrm{a}}$, J.D. Mulligan ${ }^{\mathrm{a}}$, M. \\ Oliver $^{\mathrm{a}}$, J. Schambach ${ }^{\mathrm{b}}$, N. Smirnov ${ }^{\mathrm{a}}$ \\ ${ }^{a}$ Yale University, New Haven, CT, USA \\ ${ }^{b}$ University of Texas at Austin, Austin, TX, USA
}

\begin{abstract}
We measured the properties of a novel combination of two Gas Electron Multipliers with a Micromegas for use as amplification devices in high-rate gaseous time projection chambers. The goal of this design is to minimize the buildup of space charge in the drift volume of such detectors in order to eliminate the standard gating grid and its resultant dead time, while preserving good tracking and particle identification performance. To characterize this micro-pattern gas detector configuration, we measured the positive ion back-flow and energy resolution at various element gains and electric fields, using a variety of gases, and additionally studied crosstalk effects and discharge rates. At a gain of 2000, this configuration achieves an ion back-flow below $0.4 \%$ and an energy resolution better than $\sigma / \mathrm{E}=12 \%$ for ${ }^{55} \mathrm{Fe} \mathrm{X}$-rays.
\end{abstract}

Keywords: GEM, Micromegas, Micro-pattern gas detector, Time projection chamber

\section{Introduction}

A critical issue for time projection chamber (TPC) detectors is space charge distortion (SCD) due to the accumulation of positive ions in the TPC drift volume [1]. This arises primarily from the ion back-flow (IBF) of positive ions from the gas amplification region, along with a contribution from primary ionization (from charged particles traversing the gas volume). Slow-moving positive ions distort the electric field uniformity and consequently distort the ionization electron drift trajectories, even for perfect external electric and magnetic field alignment and small transverse diffusion of the gas mixture.

The contribution of the primary ionization to the SCD can be minimized by two approaches. First, one can increase the electric field in the TPC drift volume, as ion drift speed is approximately proportional to the electric field.

Email address: nikolai.smirnov@yale.edu (N. Smirnov) 
Second, one can select a gas mixture to decrease the primary ionization itself, and to increase the ion mobility 2 .

To minimize IBF, wire grid structures called gating grids (GGs) have traditionally been used [3. In the open state, GGs have a high transparency for ionization electrons to pass through to the gas amplification unit, typically a multi-wire proportional chamber. The GG can then be closed to collect ions from the gas amplification (gain) step. As a result, the IBF due to the gas amplification is very low. However, since the GG must remain closed until the positive ions from the avalanche at the anode wire have drifted to the grid, the TPC has an intrinsic dead time that limits the readout rate. Also, since the GG is a triggered element, there is a loss of track information near the readout planes during the time it takes to trigger and open the grid.

For current experiments employing large TPCs (e.g. STAR, ALICE) and those of the future, it is desirable to find a solution to minimize dead time by eliminating the GG or perhaps using a modified GG structure 4, 5. The challenge is to minimize IBF from the gas amplification region to a level acceptable from the perspective of distortion corrections, such that track reconstruction and analysis have comparable performance to a GG solution [6, 7]. One possible solution is to use micro-pattern gas detectors (MPGDs), which have intrinsically low IBF. In particular, multi-layer MPGDs are promising candidates, as a stack of such elements allows multiple IBF-suppressing layers as well as flexibility in operational voltages and alignment, with only a small loss in electron transparency [8, 9]. Simulations for the ALICE TPC [10] have shown that at the foreseen gain of $2000\left(\mathrm{Ne}+\mathrm{CO}_{2}+\mathrm{N}_{2}(90-10-5)^{1}\right)$, with IBF as high as $2 \%$ and energy resolution of $14 \%(\sigma / \mathrm{E})$ or better (for ${ }^{55} \mathrm{Fe}$ X-rays), TPC SCD can be corrected to an acceptable level in terms of TPC track finding, PID capability, and momentum resolution. In this paper, we report our investigation of the performance of a gain configuration for TPC gas amplification using two Gas Electron Multipliers (GEMs) [11] plus a Micromegas (MMG) [12] in terms of $\mathrm{IBF}$, energy resolution, and stability.

\section{Experimental Technique}

\subsection{Operating Principles}

Figure 1 illustrates the 2-GEM + MMG setup used for these studies and defines the various elements and fields. The foremost operating principle is that the MMG provides most of the gain while the GEMs pre-amplify the signal for the MMG, so that it can be run at a relatively low voltage in order to reduce its discharge probability [13. In addition, the GEMs help spread ionization electrons through diffusion and hole pattern misalignment so that a particularly dense cluster is less likely to cause a discharge in the MMG; it has been demonstrated both in measurements [14] and simulation [15] that additional electron

\footnotetext{
${ }^{1}$ This notation reports the relative proportions of each gas in the mixture.
} 


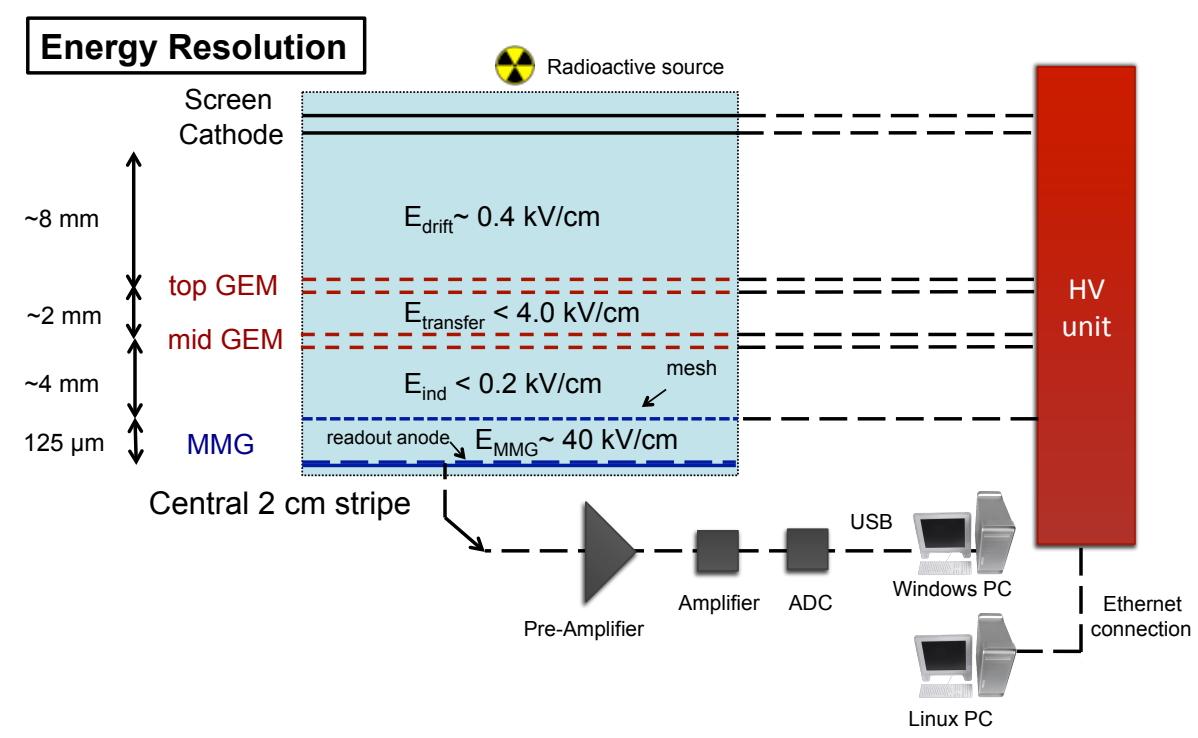

(a) Energy resolution measurement setup.

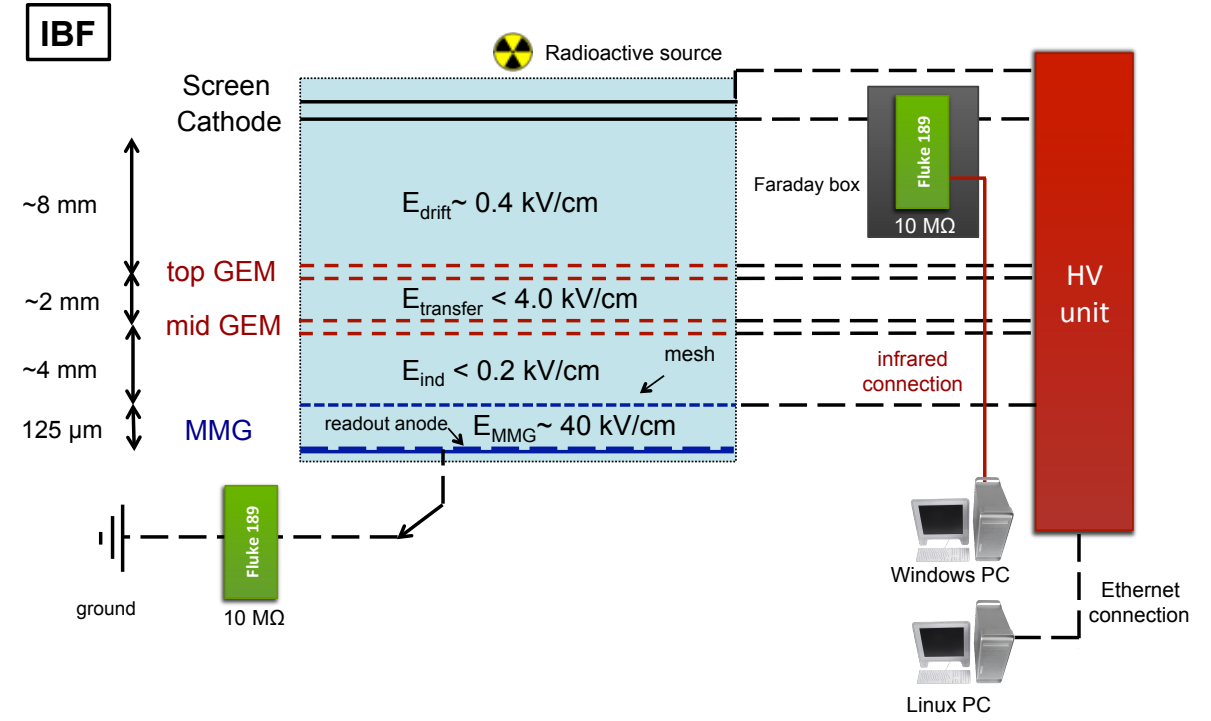

(b) IBF measurement setup.

Figure 1: Experimental setup for a chamber with two stacked GEM foils and one MMG. The listed electric fields are the nominal values.

53 spread above the MMG mesh reduces the discharge rate and can improve track 54 or ionization cluster spatial reconstruction precision.

The goal is then to tune the gains and fields in order to reduce IBF and increase energy resolution. The optimum effective gain of the GEMs is a com- 


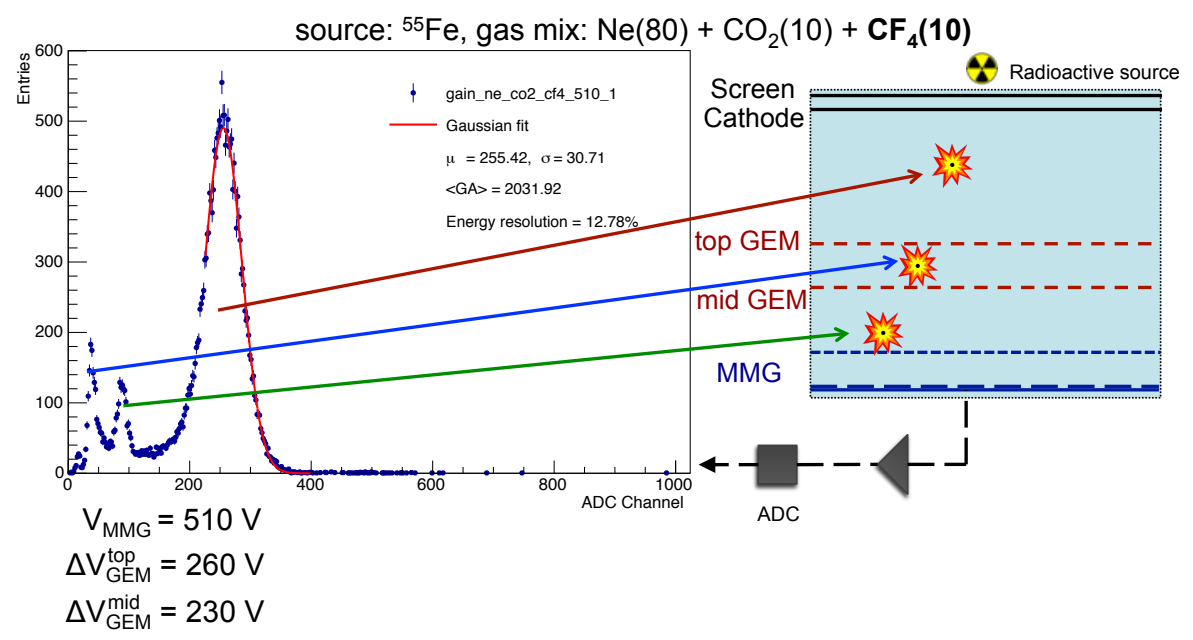

Figure 2: An example of the ${ }^{55} \mathrm{Fe}$ spectra showing the correspondence between the location of an X-ray absorption and each peak.

promise between better energy resolution, which would favor higher gain, and lower IBF from the GEMs, which would favor lower gain; the IBF contributed by a single GEM can be as much as $20 \%$ of the ionization it produces. The top GEM is particularly sensitive to this trade-off, as it is the first gain element in the stack. Note that the effective GEM gain (total charge exiting the GEM divided by total charge drifting to the GEM) is a function of the voltage across the GEM, as well as the electric fields above and below the GEM [6]. The IBF of the MMG scales with the ratio of the induction field to the MMG amplification field, $\mathrm{E}_{\text {ind }} / \mathrm{E}_{\mathrm{MMG}}$ [7, so the induction field is typically kept as low as possible. The primary purpose of the mid GEM is therefore to transfer electrons from the strong field in the transfer gap between the GEM foils to the lower field in the induction gap above the MMG. Accordingly, we operated the mid GEM with an effective gain less than 1 . This feature can be seen clearly in the example spectrum shown in Fig. 2. In addition to tuning the voltages, the IBF can be further suppressed by arranging the GEM hole patterns to assure maximum mis-alignment. The top GEM foil was rotated by $90^{\circ}$ relative to the mid GEM to increase the hole mis-alignment.

\subsection{Outline of Measurements}

This paper focuses on measurements of energy resolution and IBF under a variety of conditions in order to optimize performance of this detector design. As a first step, we varied the electric fields between the elements $\left(E_{\text {drift }}, E_{\text {transfer }}\right.$, and $\mathrm{E}_{\text {ind }}$ ) in order to find reasonable operating points. Using these field configurations, we then proceeded to our main measurement: a parameter scan of energy resolution vs. IBF by varying the voltages of each MPGD element 
$\left(\Delta \mathrm{V}_{\mathrm{GEM}}^{\mathrm{top}}, \Delta \mathrm{V}_{\mathrm{GEM}}^{\mathrm{mid}}\right.$, and $\left.\mathrm{V}_{\mathrm{MMG}}\right)$. We measured the performance using a variety of gas mixtures, with argon and neon as the primary gases, and with $\mathrm{CO}_{2}, \mathrm{~N}_{2}$, $\mathrm{CH}_{4}$, and $\mathrm{CF}_{4}$ as additional components. Finally, we further characterized this detector design by investigating the MMG crosstalk effect and MMG E-field uniformity, as well as the discharge rate.

\subsection{Measurement Procedure}

We conducted measurements with several small $10 \times 10 \mathrm{~cm}^{2}$ chambers with a variety of readout plane geometries. We operated with a gas amplification 1500-2500, typical for a TPC readout in order to maintain a good signal to noise ratio with a reasonable electronic dynamic signal range for $\mathrm{dE} / \mathrm{dx}$ measurements. There are two typical electronics configurations for these chambers. Fig. 1a shows the experimental setup for energy resolution measurements with the anode connected to a pre-amp/shaper amp/ADC chain. We measured the energy resolution as $\sigma / \mathrm{E}$ of ${ }^{55} \mathrm{Fe} \mathrm{X}$-rays. To minimize electronic noise, we connected a small central section of the anode $\left(\approx 20 \mathrm{~cm}^{2}\right)$ to the readout electronics, with the rest of the anode grounded. We used the ${ }^{55} \mathrm{Fe} \mathrm{X}$-ray response in this configuration to set the chamber gain. Figure $1 \mathrm{~b}$ shows the setup for IBF measurements, where we connected the cathode to a high voltage source through a floating picoammeter while the anode was connected to a similar meter.

We used standard GEM foils with $140 \mu \mathrm{m}$ pitch and $70 \mu \mathrm{m}$ (copper) / $50 \mu \mathrm{m}$ (apical) diameter produced by CERN ${ }^{2}$ and Tech-Etch ${ }^{3}$ MMG detectors were prepared at CERN ${ }^{4}$ and CEA Saclay Lab (CEA/IRFU/SEDI) using bulk technology, with a 400 line-per-inch mesh positioned $126 \mu \mathrm{m}$ from the readout plane. Each GEM foil was tested for leakage current at HV as well as gain uniformity.

We maintained rather low operating voltages for all three gain elements and observed no discharges during these measurements. However, in a longer term experiment, discharges will occur. Thus, we performed all measurements with a discharge protection network at the preamplifier input, and took additional data (reported below) to estimate the discharge rate using both laboratory sources and a high intensity hadron beam.

To characterize the performance with a given gas mixture, we first calibrated electronic gain by using a known capacitor and voltage step to inject a known charge into the preamplifier input. Then we took ${ }^{55} \mathrm{Fe}$ spectra for several values of $\mathrm{V}_{\mathrm{MMG}}$. At each MMG setting, we tuned the GEM voltages to set the total chamber gain to $\approx 2000$, and measured the energy resolution $\left({ }^{55} \mathrm{Fe}\right.$ peak $\left.\sigma / \mathrm{E}\right)$. For each set of voltages, we then used an intense ${ }^{90} \mathrm{Sr}$ source to measure the anode and cathode currents to calculate the IBF. For this measurement, we adjusted the source intensity to keep the anode current below $100 \mathrm{nA}$ to avoid saturation from ion buildup in the chamber.

\footnotetext{
${ }^{2}$ http://cern.ch/gem.

${ }^{3}$ Tech-Etch Inc, 45 Aldrin Rd, Plymouth, MA, USA.

${ }^{4}$ https://ep-dep-dt.web.cern.ch/micro-pattern-technologies. The first MMG was provided courtesy of L. Ropelewski, RD51, CERN.
} 
Since the IBF currents are quite small, we took care to avoid noise and account for all current sources. As seen in Fig. 1b, we placed a screen electrode just outside the chamber cathode. The screen was operated at the same voltage as the cathode and collects any ions produced outside the chamber. The picoammeter measuring the cathode current was placed in a shielded enclosure to avoid pickup noise and was read out by an infrared link to a computer. For each set of voltages, we measured the anode and cathode currents. In addition, we biased the chamber to measure the cathode current from the initial ionization in the drift gap and the anode current from ionization in the MMG induction gap. We checked the gain by approximating

$$
\langle\mathrm{GA}\rangle \approx \frac{\mathrm{I}_{\text {anode }}-\left(\mathrm{I}_{\text {anode }} \text { from ionization in induction gap }\right)}{\mathrm{I}_{\text {cathode }} \text { from ionization in drift gap }} .
$$

The IBF fraction for each voltage setting is then calculated as:

$$
\mathrm{IBF}=\frac{\mathrm{I}_{\text {cathode }}-\left(\mathrm{I}_{\text {cathode }} \text { from ionization in drift gap }\right)}{\mathrm{I}_{\text {anode }}-\left(\mathrm{I}_{\text {anode }} \text { from ionization in induction gap }\right)} .
$$

The precision of all measurements is $10-15 \%$ for IBF and $3-5 \%$ for energy resolution. For IBF measurements, the dominant uncertainty was due to pickup noise on the picoammeter. For energy resolution measurements, the dominant uncertainty was systematic uncertainty in setting the fitting range in the Gaussian fitting procedure for the ${ }^{55} \mathrm{Fe}$ peak. Systematic uncertainties and repeatability were checked by comparison of measurement results for three different setups where the GEM foils, MMG, and current readout instrumentation were changed. Parameters in our laboratory such as pressure, temperature, and gas quality were monitored and controlled; for all measurements, the maximum water and oxygen content in the exit gas were $200 \mathrm{ppm}$ and $30 \mathrm{ppm}$, respectively, coming mainly from diffusion through the thin window in the chamber vessel. Additionally, after applying high voltage or after radioactive sources were introduced or changed, we waited 15-20 minutes to be sure that the detector response was stable.

\section{Results}

\subsection{E-Field scans}

Our first measurement characterized the detector performance as a function of $E_{\mathrm{drift}}, \mathrm{E}_{\text {transfer }}$, and $\mathrm{E}_{\text {ind }}$; we performed field scans for each of the three fields in a $\mathrm{Ne}+\mathrm{CO}_{2}(90-10)$ gas mixture.

First, we varied $\mathrm{E}_{\text {drift }}$ by changing the cathode voltage while keeping $\mathrm{E}_{\text {transfer }}$ and $E_{\text {ind }}$ constant. As $E_{\text {drift }}$ increases, ions back-flowing from the top GEM are more likely to escape to the drift region. That is, the ion extraction efficiency increases. The IBF therefore depends almost linearly on $\mathrm{E}_{\text {drift }}$; doubling the field approximately doubles the IBF (Fig. 3, bottom panel). The anode current remains approximately constant as $\mathrm{E}_{\mathrm{drift}}$ increases; it is plotted to emphasize that the IBF trend is not due to the small change in gain, but indeed due to 


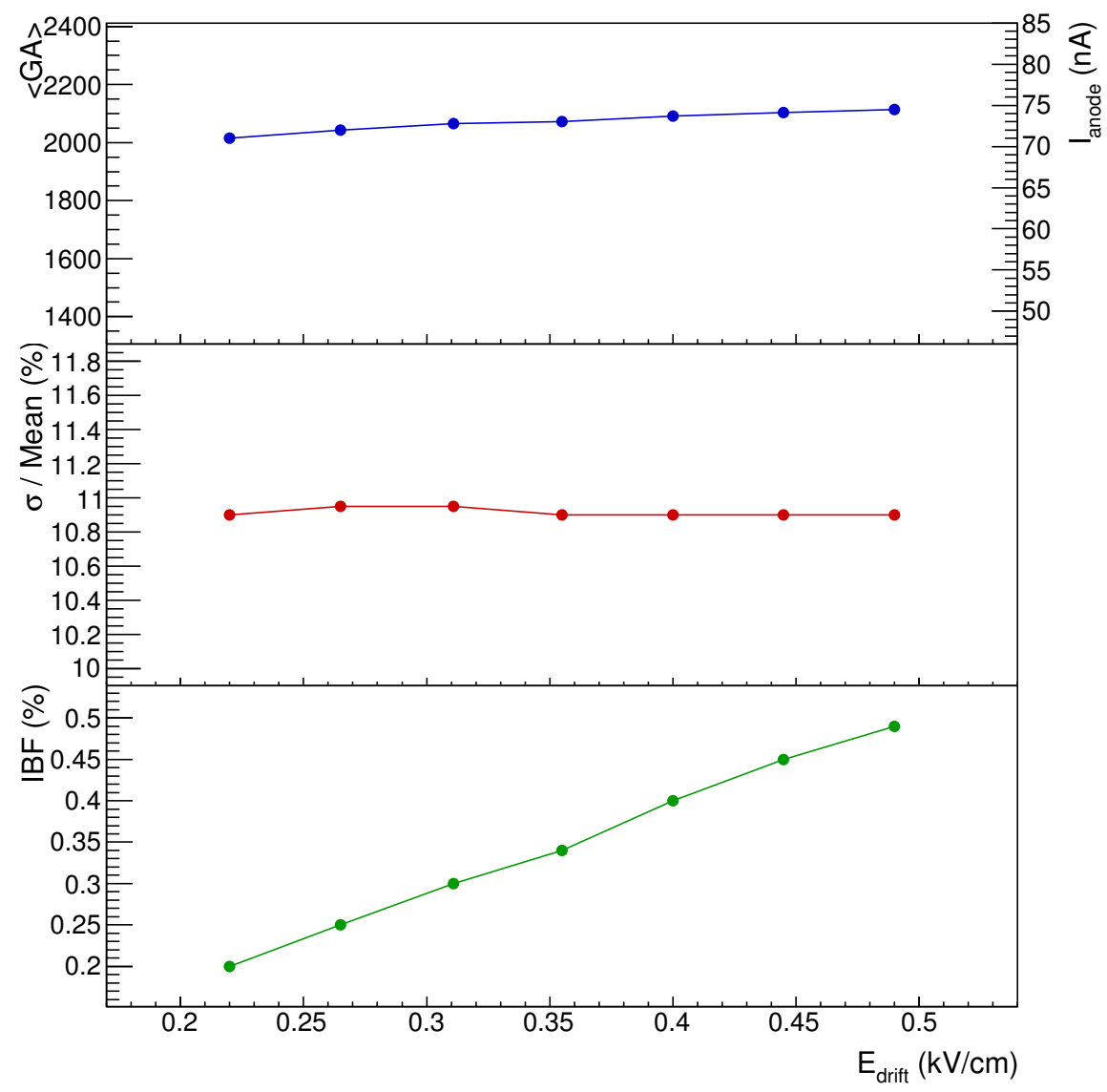

Figure 3: Scan of $\mathrm{E}_{\mathrm{drift}}$ for $\mathrm{Ne}+\mathrm{CO}_{2}(90-10), \mathrm{V}_{\mathrm{MMG}}=400 \mathrm{~V}, \Delta \mathrm{V}_{\mathrm{GEM}}^{\mathrm{top}}=242 \mathrm{~V}, \Delta \mathrm{V}_{\mathrm{GEM}}^{\mathrm{mid}}$ $=185 \mathrm{~V}, \mathrm{E}_{\text {transfer }}=2.0 \mathrm{kV} / \mathrm{cm}, \mathrm{E}_{\text {ind }}=0.075 \mathrm{kV} / \mathrm{cm}$. Gain is $\approx 2100$ (corresponding to $\left.\mathrm{I}_{\text {anode }} \approx 74 \mathrm{nA}\right)$.

the changing ion extraction efficiency from the top GEM (Fig. 3. top panel). The energy resolution remains essentially constant through this scan (Fig. 3 middle panel), since the energy resolution depends weakly on the gain (the top GEM gain is large enough to not statistically limit the resolution), and the gain changes weakly with $\mathrm{E}_{\text {drift }}$ in the range studied. However, the operating point for $\mathrm{E}_{\mathrm{drift}}$ is determined more by the drift requirements of the TPC than its effect on energy resolution and IBF; we therefore operated at $\mathrm{E}_{\mathrm{drift}}=0.4 \mathrm{kV} / \mathrm{cm}$ for all subsequent measurements.

Next, we scanned $E_{\text {transfer }}$ with $E_{\text {drift }}$ and $E_{\text {ind }}$ fixed. As $E_{\text {transfer }}$ increases, the effective gain of the top GEM increases due to enhanced electron extraction 


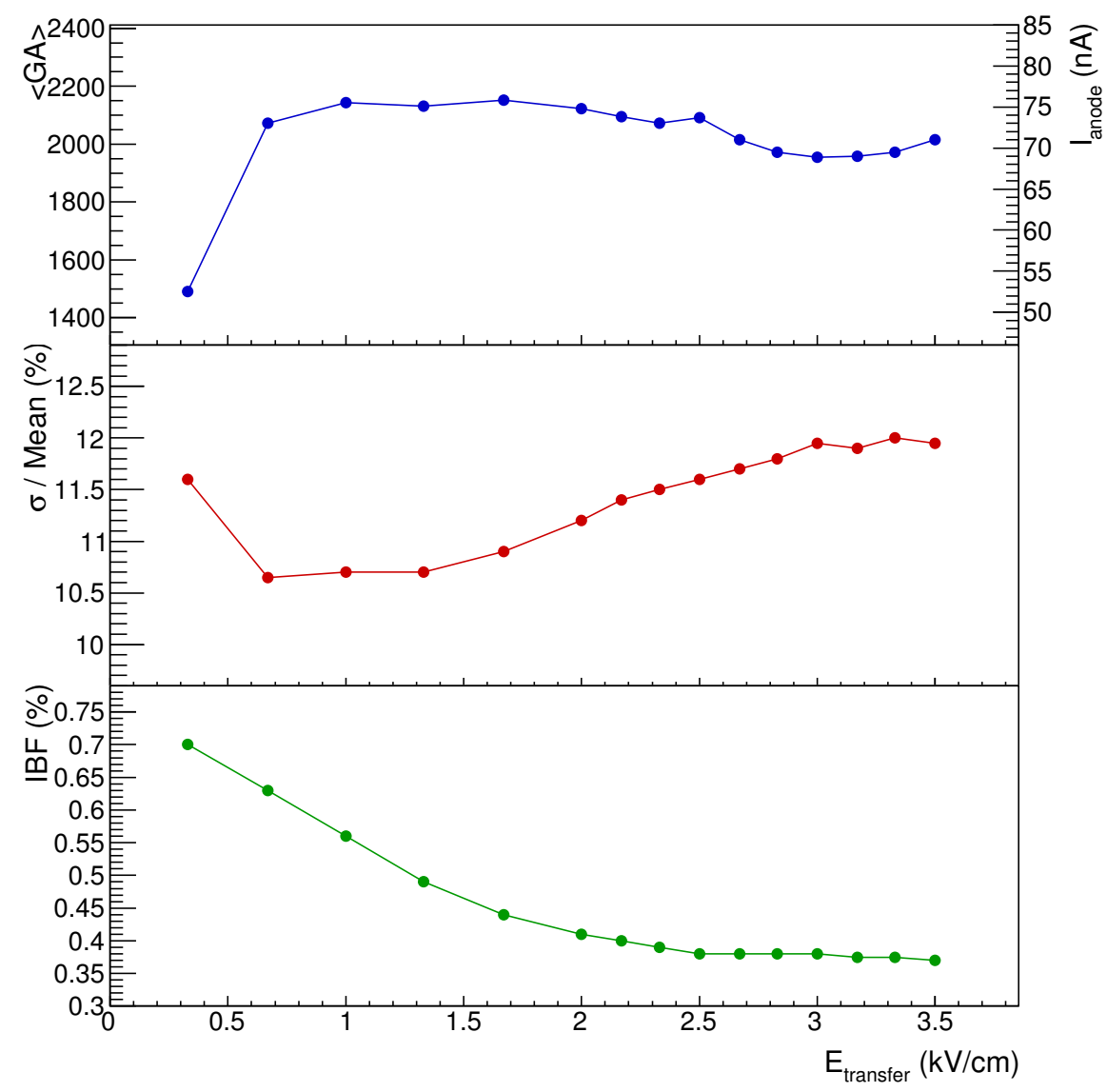

Figure 4: Scan of $\mathrm{E}_{\text {transfer }}$ for $\mathrm{Ne}+\mathrm{CO}_{2}(90-10), \mathrm{V}_{\mathrm{MMG}}=400 \mathrm{~V}, \Delta \mathrm{V}_{\mathrm{GEM}}^{\mathrm{top}}=242 \mathrm{~V}, \Delta \mathrm{V}_{\mathrm{GEM}}^{\mathrm{mid}}$ $=185 \mathrm{~V}, \mathrm{E}_{\text {drift }}=0.4 \mathrm{kV} / \mathrm{cm}, \mathrm{E}_{\text {ind }}=0.075 \mathrm{kV} / \mathrm{cm}$.

efficiency [6. This acts to improve the energy resolution, until it plateaus at $\mathrm{E}_{\text {transfer }}>1 \mathrm{kV} / \mathrm{cm}$. At the same time, the effective gain of the mid GEM decreases, which acts to degrade the energy resolution. The net effect is a balance between the behaviors of the two GEMs. The overall gain is fairly constant for $\mathrm{E}_{\text {transfer }}>1 \mathrm{kV} / \mathrm{cm}$ (Fig. 4 top panel), and the energy resolution has a small degradation due to the decreased mid GEM gain (Fig. 4 middle panel). Moreover, the IBF improves as $\mathrm{E}_{\text {transfer }}$ increases (Fig. 4, bottom panel) 6, 8. Nevertheless, within the limits $1.8<\mathrm{E}_{\text {transfer }}<3.5 \mathrm{kV} / \mathrm{cm}$, there is only weak dependence of the energy resolution and the IBF on $\mathrm{E}_{\text {transfer. }}$. Consequently, the operational $\mathrm{E}_{\text {transfer }}$ should be in this vicinity. For $\mathrm{E}_{\text {transfer }}>3.7 \mathrm{kV} / \mathrm{cm}$ 


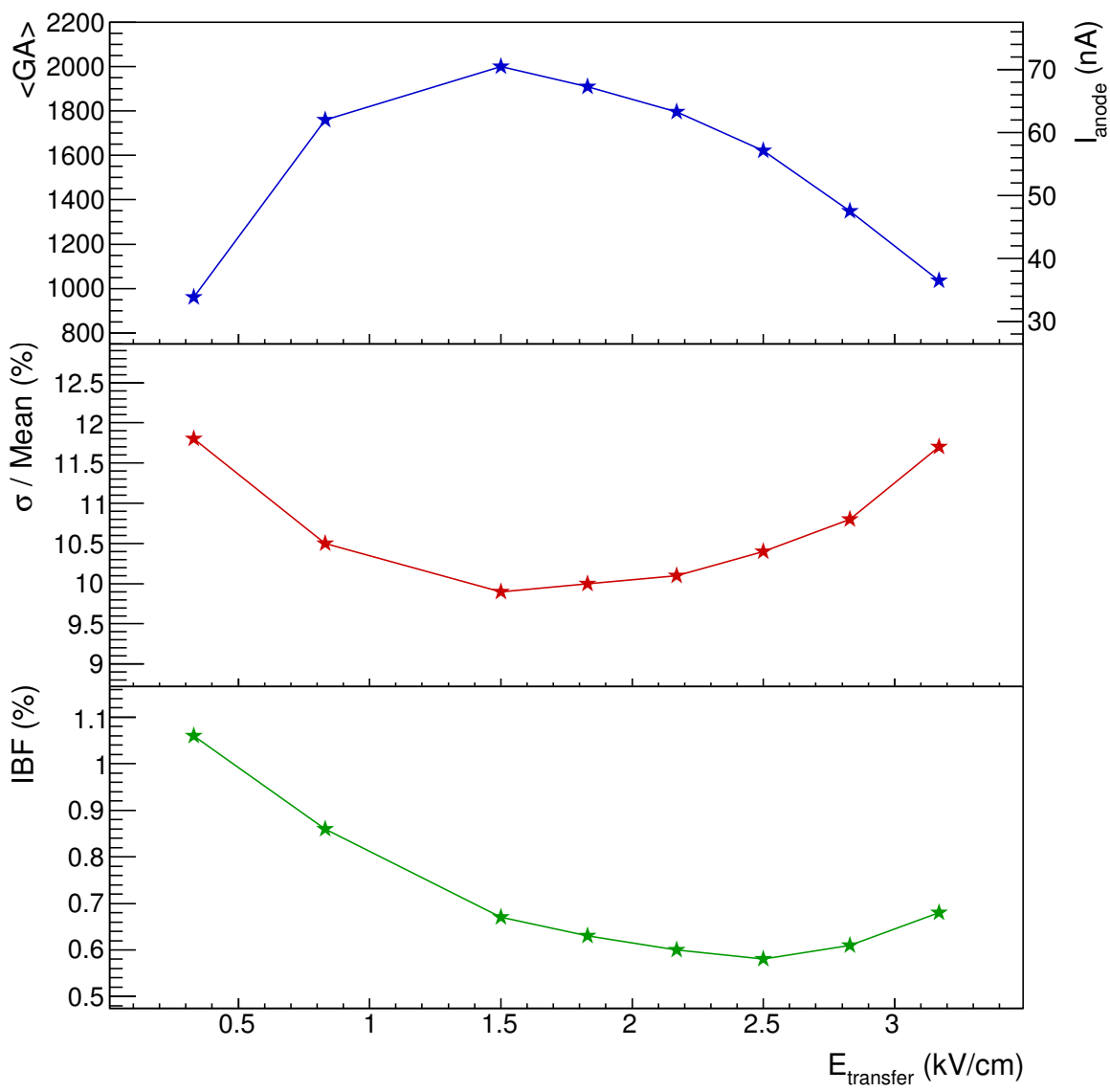

Figure 5: Scan of $\mathrm{E}_{\text {transfer }}$ for $\mathrm{Ne}+\mathrm{CO}_{2}+\mathrm{CF}_{4}(82-9-9), \mathrm{V}_{\mathrm{MMG}}=430 \mathrm{~V}, \Delta \mathrm{V}_{\mathrm{GEM}}^{\mathrm{top}}=271 \mathrm{~V}$, $\Delta \mathrm{V}_{\mathrm{GEM}}^{\mathrm{mid}}=206 \mathrm{~V}, \mathrm{E}_{\mathrm{drift}}=0.4 \mathrm{kV} / \mathrm{cm}, \mathrm{E}_{\text {ind }}=0.075 \mathrm{kV} / \mathrm{cm}$.

in $\mathrm{Ne}+\mathrm{CO}_{2}$ (90-10), gas amplification begins to occur in the transfer region, setting an upper bound for $\mathrm{E}_{\text {transfer }}$. Accordingly, in the measurements below we operated with $\mathrm{E}_{\text {transfer }} \approx 2-3 \mathrm{kV} / \mathrm{cm}$.

Gas mixtures containing $\mathrm{CF}_{4}$ have an additional constraint: for fields larger than $2.0 \mathrm{kV} / \mathrm{cm}$, the gain decreases substantially due to resonant electron absorption by $\mathrm{CF}_{4}$ (Fig. 5). Note that in this scan the GEM voltages were not varied to keep the gain fixed. To avoid this absorption effect, we used $\mathrm{E}_{\text {transfer }}$ $=1.5 \mathrm{kV} / \mathrm{cm}$.

Finally, we scanned $\mathrm{E}_{\text {ind }}$ by fixing $\mathrm{E}_{\mathrm{drift}}$ and $\mathrm{E}_{\text {transfer }}$ (as well as $\mathrm{V}_{\mathrm{MMG}}$ ), and tuning the GEM voltages to preserve the gain $\approx 2000$. Similar to the case of 


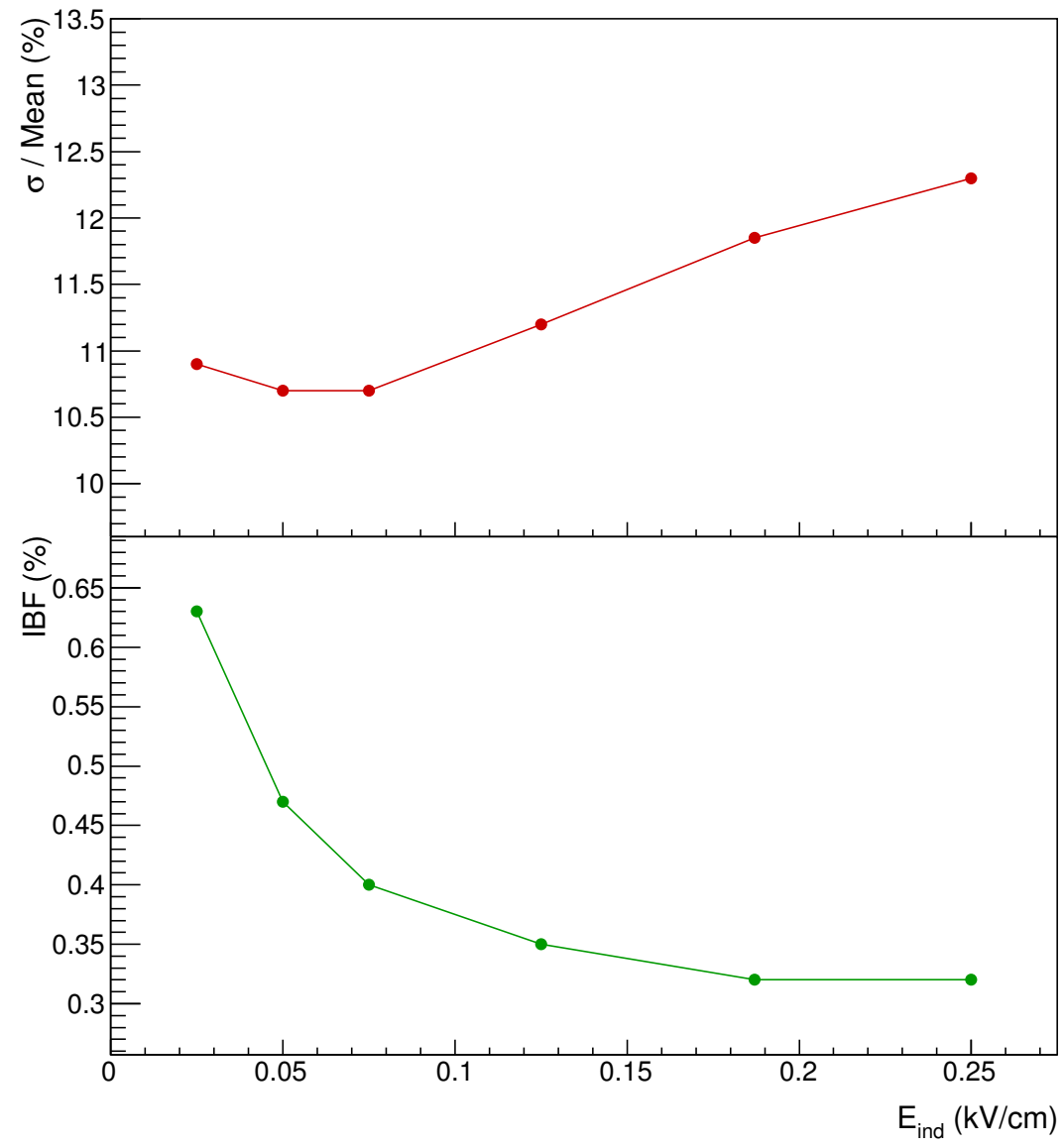

Figure 6: Scan of $\mathrm{E}_{\text {ind }}$ for $\mathrm{Ne}+\mathrm{CO}_{2}(90-10), \mathrm{V}_{\mathrm{MMG}}=400 \mathrm{~V}, \mathrm{E}_{\text {drift }}=0.4 \mathrm{kV} / \mathrm{cm}, \mathrm{E}_{\text {transfer }}$ $=2.0 \mathrm{kV} / \mathrm{cm} . \Delta \mathrm{V}_{\mathrm{GEM}}^{\mathrm{top}}$ and $\Delta \mathrm{V}_{\mathrm{GEM}}^{\mathrm{mid}}$ are tuned to keep the overall gain $\approx 2000$.

the $E_{\text {transfer }}$ scan, increasing $E_{\text {ind }}$ increases the electron extraction from the mid GEM, which increases the gain. The GEM voltages are decreased accordingly to keep the gain constant. In particular, as $E_{\text {ind }}$ increases, decreasing the top GEM gain results in a degradation of the energy resolution, and a decrease of the IBF (Fig. 6). We chose to work with $\mathrm{E}_{\text {ind }}=0.075 \mathrm{kV} / \mathrm{cm}$ in all subsequent measurements.

\subsection{Energy Resolution vs. IBF}

Next, we studied how to optimally distribute gain through the three elements in terms of maintaining good energy resolution and IBF. We changed 


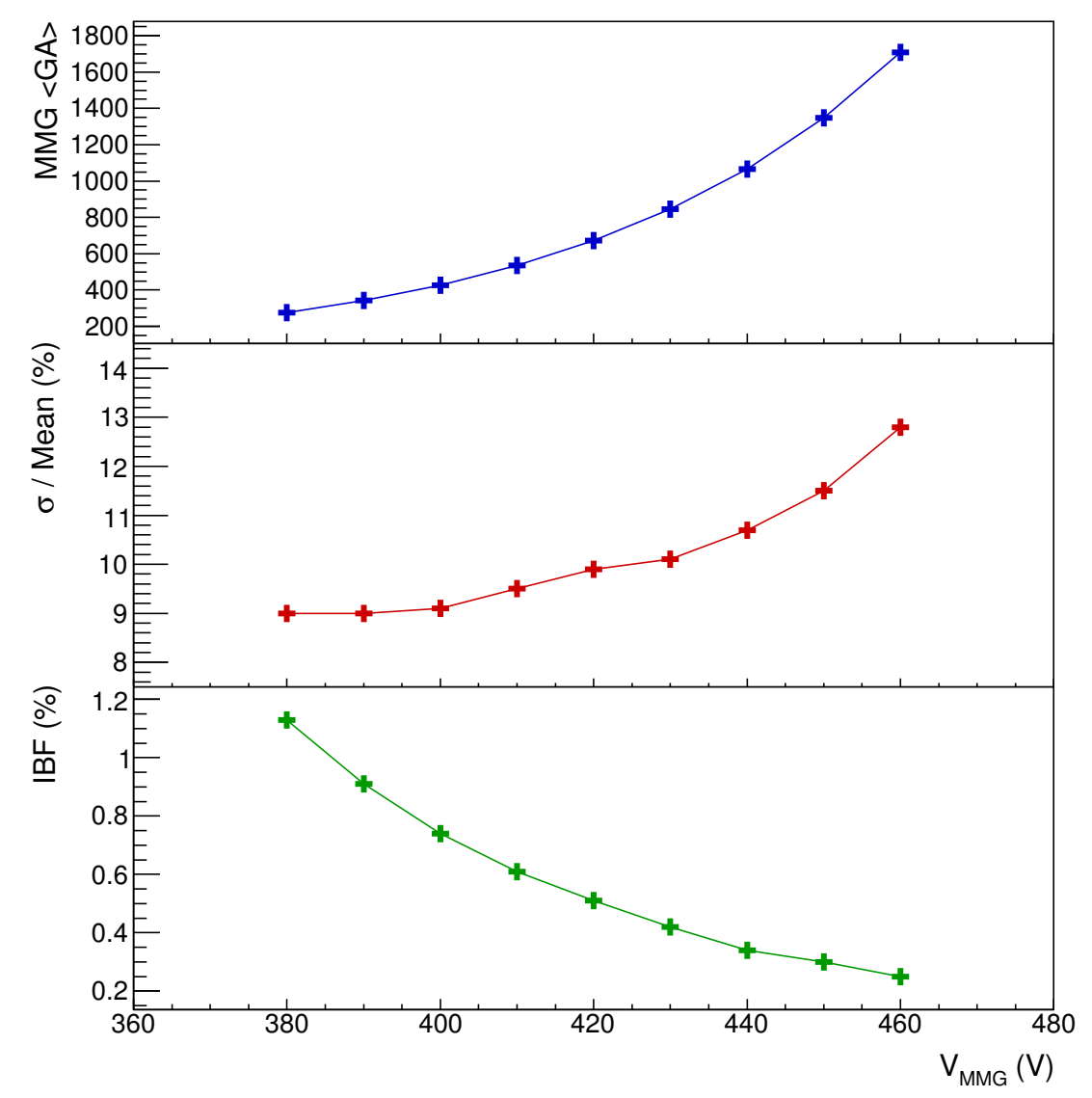

Figure 7: $\mathrm{MMG}$ gain, energy resolution, and IBF as a function of the $\mathrm{V}_{\mathrm{MMG}}$ for $\mathrm{Ne}+\mathrm{CO}_{2}+\mathrm{CH}_{4}(82-9-9), \mathrm{E}_{\text {drift }}=0.4 \mathrm{kV} / \mathrm{cm}, \mathrm{E}_{\text {transfer }}=3.0 \mathrm{kV} / \mathrm{cm}$ and $\mathrm{E}_{\text {ind }}=0.075 \mathrm{kV} / \mathrm{cm}$. $\Delta \mathrm{V}_{\mathrm{GEM}}^{\mathrm{top}}$ and $\Delta \mathrm{V}_{\mathrm{GEM}}^{\mathrm{mid}}$ are tuned to keep the overall gain $\approx 2100$.

$\mathrm{V}_{\mathrm{MMG}}$ in steps of $10 \mathrm{~V}$, starting at a voltage corresponding to a MMG gain of 200 , and then tuned the GEM voltages to preserve the overall gain of about 2000. Throughout the measurements, we fixed $E_{\text {drift }}, E_{\text {transfer, }}$, and $E_{\text {ind }}$ at $0.4,3.0$, and $0.075 \mathrm{kV} / \mathrm{cm}$, respectively. Fig. 7 illustrates the results of such a set of measurements. As discussed previously, when the MMG gain is smaller (with correspondingly higher gain in the GEMs), the energy resolution improves (Fig. 7, middle panel) at the expense of a higher IBF (Fig. 7, bottom panel). At the other extreme, when the gain is almost entirely provided by the MMG, IBF improves at the expense of worse energy resolution. Thus, the IBF and energy resolution anti-correlate with each other when the gas amplification share of 
each gain element is varied.

In order to optimize the performance of the system, we examined these scans in the 2D phase space of energy resolution and IBF. Figure 8 shows the result for a $\mathrm{Ne}+\mathrm{CO}_{2}(90-10)$ gas mixture. Energy resolution vs. IBF curves are shown for various fixed $\Delta \mathrm{V}_{\mathrm{GEM}}^{\mathrm{mid}}$; we scanned $\mathrm{V}_{\mathrm{MMG}}$ with $\Delta \mathrm{V}_{\mathrm{GEM}}^{\mathrm{top}}$ tuned as necessary to keep the overall gain fixed at about 2000. The result of this procedure defines a curve in this $2 \mathrm{D}$ space, for each fixed $\Delta \mathrm{V}_{\mathrm{GEM}}^{\mathrm{mid}}$. While there is not a large difference in performance, there is a slight preference for lower $\Delta \mathrm{V}_{\mathrm{GEM}}^{\mathrm{mid}}$.

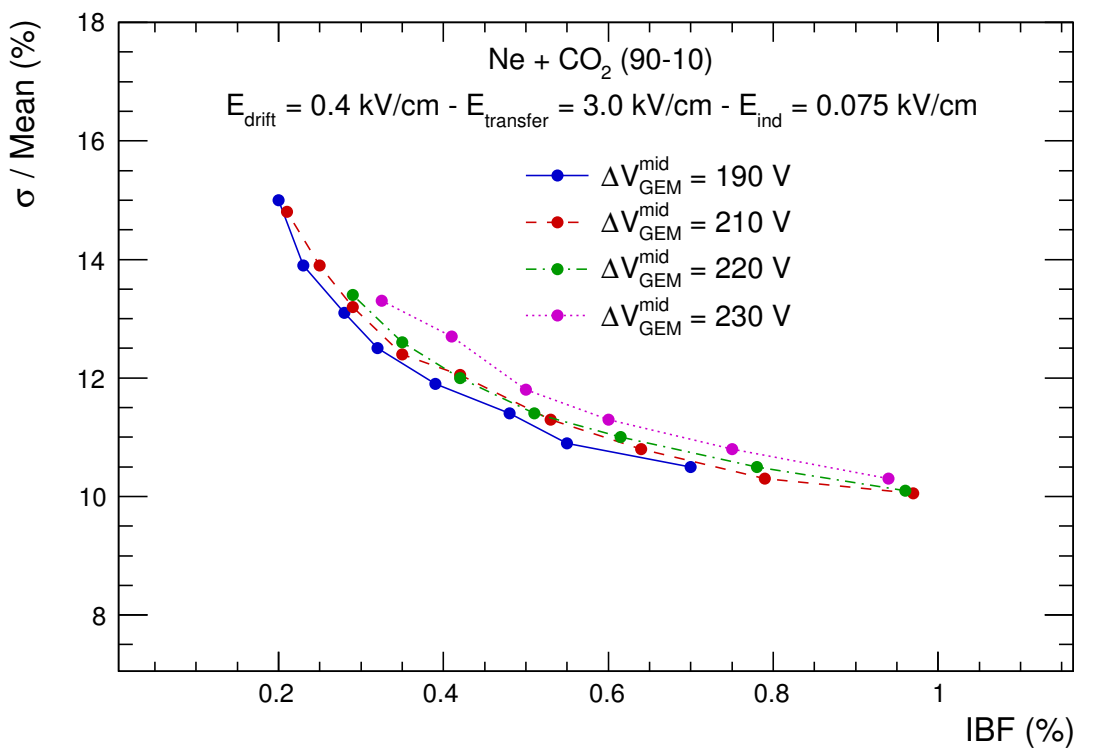

Figure 8: Energy resolution vs. IBF, varying $\mathrm{V}_{\mathrm{MMG}}$ and $\Delta \mathrm{V}_{\mathrm{GEM}}^{\mathrm{top}}$ for several fixed values of $\Delta \mathrm{V}_{\mathrm{GEM}}^{\mathrm{mid}}$.

\begin{tabular}{llll}
\hline Gain & IBF $(\%)$ & $\epsilon$ & Gas Mixture \\
\hline 2000 & 0.3 & 6.0 & $\mathrm{Ar}+\mathrm{CO}_{2}(90-10)$ \\
3000 & 0.21 & 6.3 & $\mathrm{Ar}+\mathrm{CO}_{2}(90-10)$ \\
5500 & 0.11 & 6.5 & $\mathrm{Ar}+\mathrm{CO}_{2}(70-30)$ \\
\hline
\end{tabular}

Table 1: Values of $\epsilon \equiv \mathrm{IBF} \times$ gain extracted from the measurement of the gain and IBF with energy resolution fixed at $12 \%$ along with the $E_{\text {drift }}, E_{\text {transfer }}$, and $E_{\text {ind }}$ fixed. $\Delta V_{G E M}^{\text {top }}$ and $\Delta \mathrm{V}_{\mathrm{GEM}}^{\mathrm{mid}}$ were approximately the same, although the precise setup and foils varied.

We performed the same energy resolution vs. IBF measurements for a variety of argon and neon based gas mixtures. Figure 9a shows a comparison between $\mathrm{CH}_{4}$ and $\mathrm{CO}_{2}$ in argon. The $\mathrm{CO}_{2}$ mixture exhibits better energy resolution at 


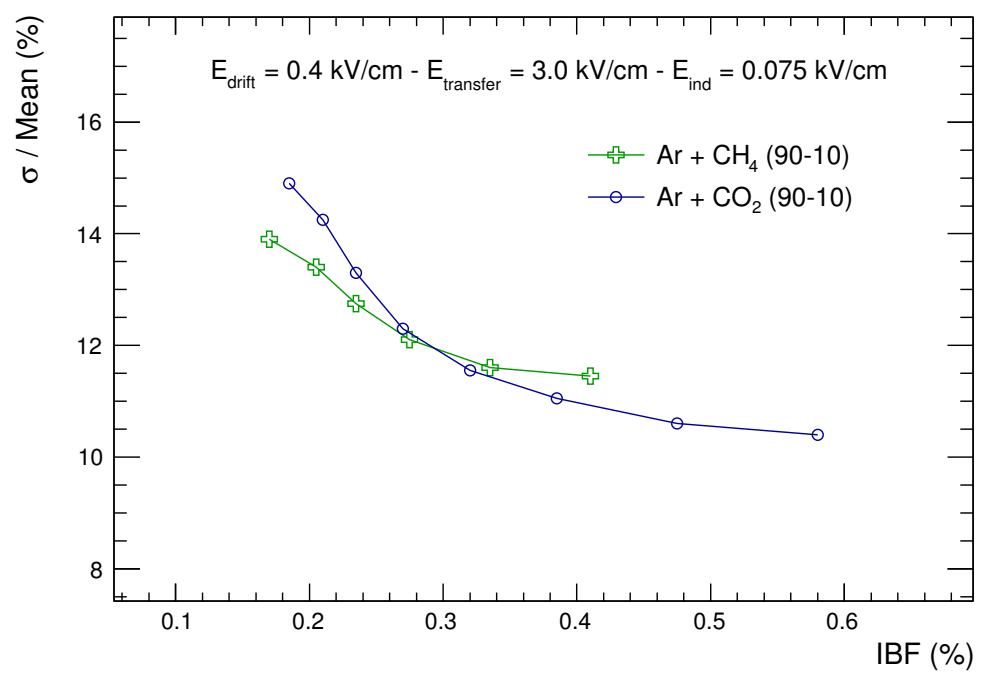

(a) Argon-based gas mixtures.

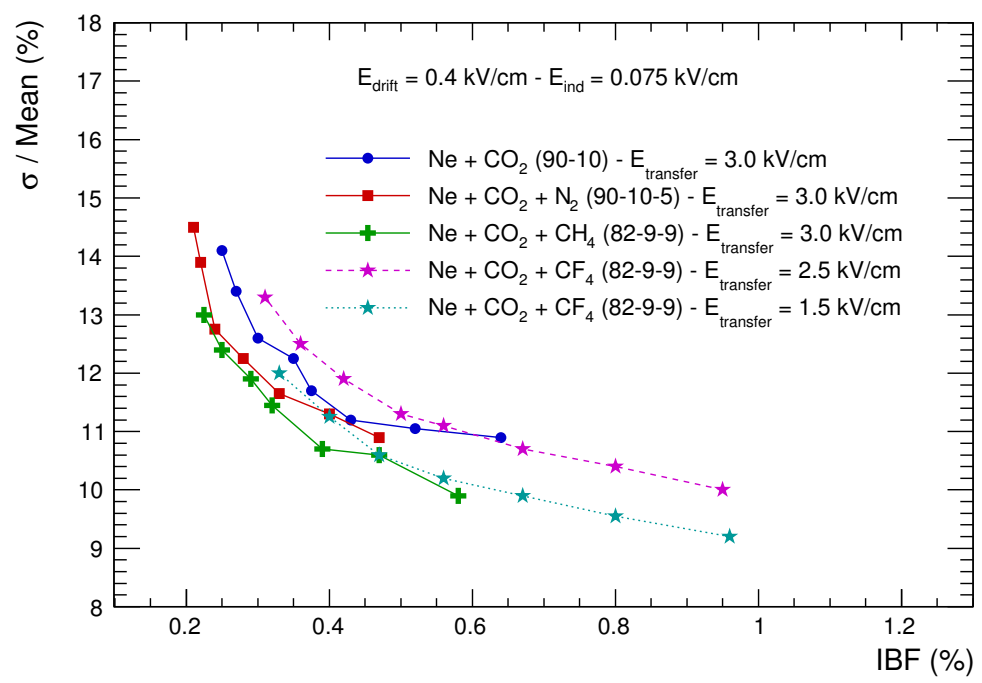

(b) Neon-based gas mixtures.

Figure 9: Energy resolution vs. IBF for a chamber with two stacked GEM foils and one MMG, filled with several gas mixtures based on neon or argon.

slightly higher IBF. Figure 9b shows a comparison between a number of different gases added to the baseline $\mathrm{Ne}+\mathrm{CO}_{2}$ mixture. Note that $\mathrm{E}_{\text {transfer }}$ is lower for $\mathrm{CF}_{4}$ to avoid the electron capture described above. These measurements 
seem to suggest a slight preference for $\mathrm{Ne}+\mathrm{CO}_{2}+\mathrm{CH}_{4}$, taking into account the ion mobility and quenching properties (to reduce discharge) of $\mathrm{CH}_{4}$. However, voltage configurations haven't been fully optimized for these gas mixtures; a more detailed investigation should be done for any gas mixture selected as a working TPC gas.

Note that when designing a TPC, the relevant parameter determining SCD is the ion density in the main TPC drift volume, which depends not just on IBF but also on other parameters such as the ion mobility and the level of primary ionization. The curves from Fig. 8 and Fig. 9 should be interpreted accordingly; despite similar IBF curves in neon and argon, an argon-based gas mixture would result in higher space-charge buildup, due to its $\sim 3 \times$ smaller ion mobility. In a large TPC, it would be difficult to compensate for this difference, since it would require a large increase in the cathode voltage.

Additionally, we performed the same energy resolution vs. IBF measurements with $\mathrm{Ar}+\mathrm{CO}_{2}$ gas mixtures, but changing the overall gain (mainly by changing the MMG gain) in the range suitable for a TPC with modern electronics, in regards to signal-to-noise and signal dynamic range. We demonstrated that for fixed energy resolution $\approx 12 \%$, the product $\epsilon=\mathrm{IBF} \times$ Gain varies slowly, as can be seen in Table 1. The increased ion production in the MMG as its gain increases is approximately compensated by the increased ion capture in the $M M G$ due to the increase in the ratio of $\mathrm{E}_{\mathrm{MMG}}$ to $\mathrm{E}_{\text {ind }}$. This allows some freedom in selecting the gas amplification in order to optimize signal-to-noise.

\section{3. $M M G$ crosstalk effect and E-field uniformity}

In a high-rate environment, crosstalk between readout elements degrades the energy resolution of a detector. For a MMG detector, the mesh is quite close to the readout plane. This means that the capacitance between the mesh and the readout elements is larger than is typical for wire chambers or GEM chambers, which leads to increased capacitive coupling between readout elements and thus increased crosstalk. In a large chamber operating at a high rate, this crosstalk can degrade the energy resolution. For the chambers we tested, with a 126 micron gap and 400 lines per inch mesh, we measured a mesh to readout capacitance of $17 \mathrm{pF} / \mathrm{cm}^{2}$. In our small chamber tests, using a standard charge sensitive preamplifier and a shaping amplifier with a $2 \mu$ s shaping time, we measured an inverse polarity crosstalk amplitude of $0.35 \%$ per $\mathrm{cm}^{2}$ pad size, with the expectation that the crosstalk is proportional to the readout pad to mesh capacitance.

Another feature of MMG elements resulting from the small gap between the mesh and readout plane is the influence of the readout pattern on the energy resolution. The width of the spaces between readout elements will be a significant fraction of the gap to the mesh and will therefore cause large local variation in the electric field and hence the gain. For example, we tested a MMG with chevron style pad readout ( 6 zigzags, $4 \times 8 \mathrm{~mm}^{2}$ pads), which showed $30 \%$ worse energy resolution compared to the MMG with rectangular pads of the same size. 


\subsection{Discharge Rate}

To test the discharge behavior, we constructed a spark test chamber with spark protection on the MMG and readout plane. The chamber had a larger drift gap $(43.6 \mathrm{~mm})$, with a collimated ${ }^{241} \mathrm{Am}$ source $11.1 \mathrm{~mm}$ above a small hole in the cathode. This source could be remotely moved relative to the cathode in order to vary the rate of $\alpha$ particles in the chamber. An ${ }^{55} \mathrm{Fe}$ source was also mounted in the chamber to monitor the chamber gain. Signals from the two GEM foils and the MMG mesh were coupled through capacitors, attenuators, and discriminators to scalers to count sparks. The signal from the anode was also coupled to a scaler to count the total number of $\alpha$ particles.

We measured a discharge rate of less than $3 \times 10^{-7}$ per $\alpha$ in $\mathrm{Ne}+\mathrm{CO}_{2}(90-$ 10). With $\mathrm{Ne}+\mathrm{CO}_{2}+\mathrm{CH}_{4}(82-9-9)$, the discharge rate decreased by an order of magnitude $\left(2 \times 10^{-8}\right)$.

To further measure the discharge behavior, two $21 \times 26 \mathrm{~cm}^{2}$ detectors assembled at Yale, with MMG produced at CERN and GEM foils from the PHENIX Hadron Blind Detector [16], filled with $\mathrm{Ne}+\mathrm{CO}_{2}+\mathrm{N}_{2}(90-10-5)$, were tested by the ALICE TPC-Upgrade Collaboration in a SPS beam at CERN [17. The beam of $6 \times 10^{6} 150 \mathrm{GeV}$ pions per $4.5 \mathrm{~s}$ spill was incident on a $40 \mathrm{~cm}$ iron target just upstream of the chambers, creating a high-intensity mixed particle shower perpendicular to the pad plane. The equivalent minimum ionizing particle flux incident on the chamber was measured by calibrating the anode current of a chamber just upstream of our test chambers to the counted beam flux without the iron target. A discharge rate of $3.5 \times 10^{-10}$ per chamber particle was measured. Approximately $5 \times 10^{11}$ chamber particles were accumulated. It was also observed that the sparking rate does not change much when the GEM voltages and transfer fields are switched off, indicating that the sparking is mainly due to the interaction of beam particles with the MMG. It should be noted that the spark does not damage the MMG, but rather poses the problem of a high voltage drop (with resultant dead time) and risk for readout electronics. Work is in progress to improve spark protection, such as providing a resistive layer on the pad plane to limit the discharge, and the HV drop and its recovery time [18, 19, 20].

\section{Conclusions}

In an effort to eliminate the standard gating grid in TPCs by minimizing the buildup of space charge in the drift volume, we investigated and characterized the use of 2-GEM + MMG chambers for the TPC gas amplification region. We selected this combination of MPGDs with the intention of minimizing $\mathrm{E}_{\mathrm{ind}} / \mathrm{E}_{\mathrm{MMG}}$ independent of the TPC drift field, while keeping good energy resolution. To achieve good energy resolution, we employed a strong transfer field between the foils, and operated the top GEM with 3-5 effective gas amplification. To achieve a low induction field, the mid GEM was used to transfer electrons from the strong field to the weak field, with effective gas amplification smaller than one. With this configuration, the GEM foils provide the necessary field structure, additional IBF suppression, gain pre-amplification, and 
additional electron spread over the MMG surface. We focused on neon-based gas mixtures. In general, optimization of a zero-dead time TPC is a multiparameter problem; if the correction of SCDs is the main factor for spatial resolution and momentum reconstruction performance, neon-based gas mixtures (without isobutane) are suitable due to their large ion mobility, large TPC drift field, and small primary ionization.

We achieved simultaneously an ion back-flow below $0.4 \%$ (with $10-15 \%$ uncertainty) and an energy resolution better than $\sigma / \mathrm{E}=12 \%$ (with $3-5 \%$ uncertainty) for ${ }^{55} \mathrm{Fe} \mathrm{X}$-rays at a gain of $\approx 2000$ in a variety of gas mixtures. We reported the dependence of ion back-flow and energy resolution on the various field and amplification voltages. We also presented results on crosstalk and sparking from bench tests and with test beams. The hybrid micro-pattern gas amplification stage allows for a TPC design that can operate in a continuous mode, and serves as a viable option to limit space charge distortions in high-rate TPCs.

\section{Acknowledgments}

We acknowledge the ALICE TPC-Upgrade team for help in setting up and operating our chambers at the CERN PS and SPS beams, as well as Christina Markert (University of Texas at Austin) for help with test vessel construction and readout electronics, and the PHENIX Hadron Blind Detector collaboration for supplying GEM foils.

This work is supported by the US Department of Energy under Grant DESC004168, contract 200935 from Brookhaven National Laboratory, primary funding from US Department of Energy DE-AC02-98-CH10886, and contract 4000132727 from Oak Ridge National Laboratory, primary funding from US Department of Energy award DE-SC0014550; grants DE-SC0003892 and DESC0013391.

\section{Appendix A. Data tables}

Table Appendix A.1: Data corresponding to Fig. 3.

\begin{tabular}{rrrrrrr}
\hline \multicolumn{6}{c}{$\mathrm{V}_{\mathrm{MMG}}=400 \mathrm{~V}-\Delta \mathrm{V}_{\mathrm{GEM}}^{\mathrm{top}}=242 \mathrm{~V}-\Delta \mathrm{V}_{\mathrm{GEM}}^{\text {mid }}=185 \mathrm{~V}$} \\
\hline \multicolumn{6}{c}{ All electric fields are in units of $\mathrm{kV} / \mathrm{cm}$} \\
\hline $\mathrm{E}_{\text {drift }}$ & $\mathrm{E}_{\text {transfer }}$ & $\mathrm{E}_{\text {ind }}$ & Gain & $\mathrm{I}_{\text {anode }}(\mathrm{nA})$ & $\sigma / \mathrm{E}(\%)$ & $\mathrm{IBF}(\%)$ \\
\hline 0.220 & 2.000 & 0.075 & 2015 & 71.0 & 10.90 & 0.200 \\
0.265 & 2.000 & 0.075 & 2043 & 72.0 & 10.95 & 0.250 \\
0.311 & 2.000 & 0.075 & 2066 & 72.8 & 10.95 & 0.300 \\
0.355 & 2.000 & 0.075 & 2072 & 73.0 & 10.90 & 0.340 \\
0.400 & 2.000 & 0.075 & 2091 & 73.7 & 10.90 & 0.400 \\
0.445 & 2.000 & 0.075 & 2103 & 74.1 & 10.90 & 0.450 \\
0.490 & 2.000 & 0.075 & 2114 & 74.5 & 10.90 & 0.490 \\
\hline
\end{tabular}


Table Appendix A.2: Data corresponding to Fig. 4

\begin{tabular}{crrrrrr}
\hline \multicolumn{6}{c}{$\mathrm{V}_{\mathrm{MMG}}=400 \mathrm{~V}-\Delta \mathrm{V}_{\mathrm{GEM}}^{\text {top }}=242 \mathrm{~V}-\Delta \mathrm{V}_{\mathrm{GEM}}^{\mathrm{mid}}=185 \mathrm{~V}$} \\
\hline \multicolumn{7}{c}{ All electric fields are in units of $\mathrm{kV} / \mathrm{cm}$} \\
\hline $\mathrm{E}_{\text {drift }}$ & $\mathrm{E}_{\text {transfer }}$ & $\mathrm{E}_{\text {ind }}$ & Gain & $\mathrm{I}_{\text {anode }}(\mathrm{nA})$ & $\sigma / \mathrm{E}(\%)$ & $\mathrm{IBF}(\%)$ \\
\hline 0.400 & 0.330 & 0.075 & 1490 & 52.5 & 11.60 & 0.700 \\
0.400 & 0.670 & 0.075 & 2072 & 73.0 & 10.65 & 0.630 \\
0.400 & 1.000 & 0.075 & 2143 & 75.5 & 10.70 & 0.560 \\
0.400 & 1.330 & 0.075 & 2131 & 75.1 & 10.70 & 0.490 \\
0.400 & 1.670 & 0.075 & 2151 & 75.8 & 10.90 & 0.440 \\
0.400 & 2.000 & 0.075 & 2123 & 74.8 & 11.20 & 0.410 \\
0.400 & 2.170 & 0.075 & 2094 & 73.8 & 11.40 & 0.400 \\
0.400 & 2.330 & 0.075 & 2072 & 73.0 & 11.50 & 0.390 \\
0.400 & 2.500 & 0.075 & 2091 & 73.7 & 11.60 & 0.380 \\
0.400 & 2.670 & 0.075 & 2015 & 71.0 & 11.70 & 0.380 \\
0.400 & 2.830 & 0.075 & 1972 & 69.5 & 11.80 & 0.380 \\
0.400 & 3.000 & 0.075 & 1955 & 68.9 & 11.95 & 0.380 \\
0.400 & 3.170 & 0.075 & 1958 & 69.0 & 11.90 & 0.375 \\
0.400 & 3.330 & 0.075 & 1972 & 69.5 & 12.00 & 0.375 \\
0.400 & 3.500 & 0.075 & 2015 & 71.0 & 11.95 & 0.370 \\
\hline \multicolumn{7}{c}{}
\end{tabular}

Table Appendix A.3: Data corresponding to Fig. 5.

\begin{tabular}{rrrrrrr}
\hline \multicolumn{6}{c}{$\mathrm{V}_{\mathrm{MMG}}=430 \mathrm{~V}-\Delta \mathrm{V}_{\mathrm{GEM}}^{\text {top }}=271 \mathrm{~V}-\Delta \mathrm{V}_{\mathrm{GEM}}^{\text {mid }}=206 \mathrm{~V}$} \\
\hline $\mathrm{E}_{\text {drift }}$ & $\mathrm{E}_{\text {transfer }}$ & $\mathrm{E}_{\text {ind }}$ & Gain & $\mathrm{I}_{\text {anode }}(\mathrm{nA})$ & $\sigma / \mathrm{E}(\%)$ & $\mathrm{IBF}(\%)$ \\
\hline 0.400 & 0.330 & 0.075 & 961 & 33.9 & 11.80 & 1.060 \\
0.400 & 0.830 & 0.075 & 1760 & 62.0 & 10.50 & 0.860 \\
0.400 & 1.500 & 0.075 & 2000 & 70.5 & 9.90 & 0.670 \\
0.400 & 1.830 & 0.075 & 1909 & 67.3 & 10.00 & 0.630 \\
0.400 & 2.170 & 0.075 & 1795 & 63.3 & 10.10 & 0.600 \\
0.400 & 2.500 & 0.075 & 1620 & 57.1 & 10.40 & 0.580 \\
0.400 & 2.830 & 0.075 & 1348 & 47.5 & 10.80 & 0.610 \\
0.400 & 3.170 & 0.075 & 1035 & 36.5 & 11.70 & 0.680 \\
\hline
\end{tabular}

Table Appendix A.4: Data corresponding to Fig. 6

\begin{tabular}{|c|c|c|c|c|}
\hline \multicolumn{5}{|c|}{$\mathrm{E}_{\text {drift }}=0.4 \mathrm{kV} / \mathrm{cm}-\mathrm{E}_{\text {transfer }}=2.0 \mathrm{kV} / \mathrm{cm}-\mathrm{V}_{\mathrm{MMG}}=400 \mathrm{~V}$} \\
\hline $\mathrm{E}_{\text {ind }}(\mathrm{kV} / \mathrm{cm})$ & $\Delta \mathrm{V}_{\mathrm{GEM}}^{\mathrm{top}}(\mathrm{V})$ & $\Delta \mathrm{V}_{\mathrm{GEM}}^{\mathrm{mid}}(\mathrm{V})$ & $\sigma / \mathrm{E}(\%)$ & $\operatorname{IBF}(\%)$ \\
\hline
\end{tabular}




\begin{tabular}{lllll}
0.025 & 242 & 215 & 10.90 & 0.630 \\
0.050 & 242 & 195 & 10.70 & 0.470 \\
0.075 & 242 & 185 & 10.70 & 0.400 \\
0.125 & 242 & 171 & 11.20 & 0.350 \\
0.187 & 232 & 168 & 11.85 & 0.320 \\
0.250 & 220 & 168 & 12.30 & 0.320 \\
\hline
\end{tabular}

Table Appendix A.5: Data corresponding to Fig. 7

\begin{tabular}{rrrrrr}
\hline \multicolumn{5}{c}{$\mathrm{E}_{\text {drift }}=0.4 \mathrm{kV} / \mathrm{cm}-\mathrm{E}_{\text {transfer }}=3 \mathrm{kV} / \mathrm{cm}-\mathrm{E}_{\text {ind }}=0.075 \mathrm{kV} / \mathrm{cm}$} \\
\hline $\mathrm{V}_{\text {MMG }}(\mathrm{V})$ & $\Delta \mathrm{V}_{\mathrm{GEM}}^{\mathrm{top}}(\mathrm{V})$ & $\Delta \mathrm{V}_{\mathrm{GEM}}^{\mathrm{mid}}(\mathrm{V})$ & MMG GA & $\sigma / \mathrm{E}(\%)$ & $\mathrm{IBF}(\%)$ \\
\hline 380 & 285 & 234 & 275 & 9.00 & 1.130 \\
390 & 280 & 227 & 343 & 9.00 & 0.910 \\
400 & 275 & 221 & 427 & 9.10 & 0.740 \\
410 & 268 & 214 & 534 & 9.50 & 0.610 \\
420 & 261 & 207 & 673 & 9.90 & 0.510 \\
430 & 254 & 204 & 846 & 10.10 & 0.420 \\
440 & 246 & 200 & 1066 & 10.70 & 0.340 \\
450 & 239 & 193 & 1347 & 11.50 & 0.300 \\
460 & 230 & 192 & 1709 & 12.80 & 0.250 \\
\hline
\end{tabular}

Table Appendix A.6: Data corresponding to Fig. 8

\begin{tabular}{|c|c|c|c|c|c|}
\hline \multicolumn{6}{|c|}{$\Delta \mathrm{V}_{\mathrm{GEM}}^{\mathrm{mid}}=190 \mathrm{~V}$} \\
\hline $\mathrm{V}_{\mathrm{MMG}}(\mathrm{V})$ & $\Delta \mathrm{V}_{\mathrm{GEM}}^{\mathrm{top}}(\mathrm{V})$ & $\Delta \mathrm{V}_{\mathrm{GEM}}^{\mathrm{mid}}(\mathrm{V})$ & Gain & $\sigma / \mathrm{E}(\%)$ & $\operatorname{IBF}(\%)$ \\
\hline 360 & 255 & 190 & 1874 & 10.50 & 0.700 \\
\hline 370 & 245 & 190 & 1905 & 10.90 & 0.550 \\
\hline 380 & 235 & 190 & 1926 & 11.40 & 0.480 \\
\hline 390 & 225 & 190 & 1958 & 11.90 & 0.390 \\
\hline 400 & 215 & 190 & 1984 & 12.50 & 0.320 \\
\hline 410 & 205 & 190 & 1905 & 13.10 & 0.280 \\
\hline 420 & 195 & 190 & 1941 & 13.90 & 0.230 \\
\hline 430 & 185 & 190 & 1953 & 15.00 & 0.200 \\
\hline \multicolumn{6}{|c|}{$\Delta \mathrm{V}_{\mathrm{GEM}}^{\mathrm{mid}}=210 \mathrm{~V}$} \\
\hline $\mathrm{V}_{\mathrm{MMG}}(\mathrm{V})$ & $\Delta \mathrm{V}_{\mathrm{GEM}}^{\mathrm{top}}(\mathrm{V})$ & $\Delta \mathrm{V}_{\mathrm{GEM}}^{\mathrm{mid}}(\mathrm{V})$ & Gain & $\sigma / \mathrm{E}(\%)$ & $\operatorname{IBF}(\%)$ \\
\hline
\end{tabular}




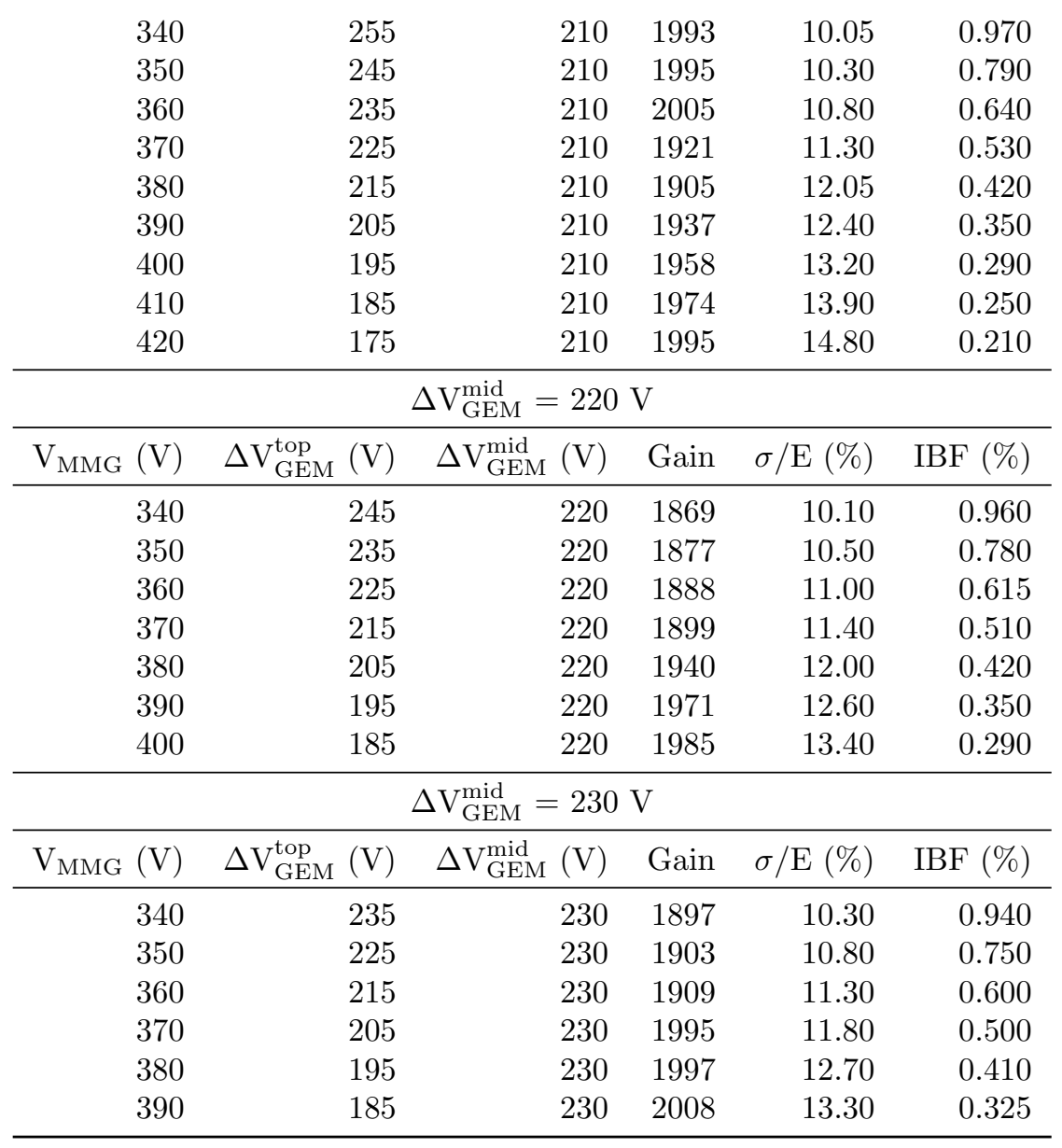

Table Appendix A.7: Data corresponding to Fig. 9a

\begin{tabular}{crrrrr}
\hline \multicolumn{6}{c}{$\mathrm{Ar}+\mathrm{CO}_{2}(90-10)$} \\
\multicolumn{6}{c}{$\mathrm{E}_{\text {drift }}=0.4 \mathrm{kV} / \mathrm{cm}-\mathrm{E}_{\text {transfer }}=3 \mathrm{kV} / \mathrm{cm}-\mathrm{E}_{\text {ind }}=0.075 \mathrm{kV} / \mathrm{cm}$} \\
\hline $\mathrm{V}_{\mathrm{MMG}}(\mathrm{V})$ & $\Delta \mathrm{V}_{\mathrm{GEM}}^{\mathrm{top}}(\mathrm{V})$ & $\Delta \mathrm{V}_{\mathrm{GEM}}^{\text {mid }}(\mathrm{V})$ & Gain & $\sigma / \mathrm{E}(\%)$ & $\mathrm{IBF}(\%)$ \\
\hline 440 & 305 & 270 & 2248 & 10.40 & 0.580 \\
450 & 295 & 265 & 2074 & 10.60 & 0.475 \\
460 & 290 & 260 & 2146 & 11.05 & 0.385 \\
470 & 280 & 255 & 2046 & 11.55 & 0.320 \\
480 & 275 & 250 & 2092 & 12.30 & 0.270 \\
490 & 270 & 245 & 2146 & 13.30 & 0.235 \\
500 & 265 & 237 & 2111 & 14.25 & 0.210 \\
510 & 260 & 229 & 2091 & 14.90 & 0.185 \\
\hline \multicolumn{5}{c}{}
\end{tabular}




\begin{tabular}{rrrrrr}
\multicolumn{7}{c}{$\mathrm{Ar}_{\mathrm{CH}}(90-10)$} \\
$\mathrm{E}_{\text {drift }}=0.4 \mathrm{kV} / \mathrm{cm}-\mathrm{E}_{\text {transfer }}=3 \mathrm{kV} / \mathrm{cm}-\mathrm{E}_{\text {ind }}=0.075 \mathrm{kV} / \mathrm{cm}$ \\
\hline $\mathrm{V}_{\mathrm{MMG}}(\mathrm{V})$ & $\Delta \mathrm{V}_{\mathrm{GEM}}^{\mathrm{top}}(\mathrm{V})$ & $\Delta \mathrm{V}_{\mathrm{GEM}}^{\text {mid }}(\mathrm{V})$ & Gain & $\sigma / \mathrm{E}(\%)$ & $\mathrm{IBF}(\%)$ \\
\hline 440 & 300 & 270 & 2175 & 11.45 & 0.410 \\
450 & 295 & 265 & 2229 & 11.60 & 0.335 \\
460 & 290 & 260 & 2320 & 12.10 & 0.275 \\
470 & 280 & 255 & 2099 & 12.75 & 0.235 \\
480 & 275 & 250 & 2168 & 13.40 & 0.205 \\
490 & 270 & 245 & 2242 & 13.90 & 0.170 \\
\hline
\end{tabular}

Table Appendix A.8: Data corresponding to Fig. 9b.

\begin{tabular}{|c|c|c|c|c|c|}
\hline \multicolumn{6}{|c|}{$\begin{array}{c}\mathrm{Ne}+\mathrm{CO}_{2}(90-10) \\
\mathrm{E}_{\text {drift }}=0.4 \mathrm{kV} / \mathrm{cm}-\mathrm{E}_{\text {transfer }}=3 \mathrm{kV} / \mathrm{cm}-\mathrm{E}_{\text {ind }}=0.075 \mathrm{kV} / \mathrm{cm}\end{array}$} \\
\hline $\mathrm{V}_{\mathrm{MMG}}(\mathrm{V})$ & $\Delta \mathrm{V}_{\mathrm{GEM}}^{\mathrm{top}}(\mathrm{V})$ & $\Delta \mathrm{V}_{\mathrm{GEM}}^{\operatorname{mid}}(\mathrm{V})$ & Gain & $\sigma / \mathrm{E}(\%)$ & $\operatorname{IBF}(\%)$ \\
\hline 360 & 235 & 210 & 2055 & 10.90 & 0.640 \\
\hline 370 & 225 & 210 & 2140 & 11.05 & 0.520 \\
\hline 380 & 220 & 205 & 2151 & 11.20 & 0.430 \\
\hline 390 & 200 & 215 & 2142 & 11.70 & 0.375 \\
\hline 400 & 195 & 210 & 2133 & 12.25 & 0.350 \\
\hline 410 & 190 & 200 & 2134 & 12.60 & 0.300 \\
\hline 420 & 195 & 185 & 2160 & 13.40 & 0.270 \\
\hline 430 & 190 & 180 & 2163 & 14.10 & 0.250 \\
\hline \multicolumn{6}{|c|}{$\begin{array}{c}\mathrm{Ne}+\mathrm{CO}_{2}+\mathrm{N}_{2}(90-10-5) \\
\mathrm{E}_{\mathrm{drift}}=0.4 \mathrm{kV} / \mathrm{cm}-\mathrm{E}_{\text {transfer }}=3 \mathrm{kV} / \mathrm{cm}-\mathrm{E}_{\text {ind }}=0.075 \mathrm{kV} / \mathrm{cm}\end{array}$} \\
\hline $\mathrm{V}_{\mathrm{MMG}}(\mathrm{V})$ & $\Delta \mathrm{V}_{\mathrm{GEM}}^{\mathrm{top}}(\mathrm{V})$ & $\Delta \mathrm{V}_{\mathrm{GEM}}^{\mathrm{mid}}(\mathrm{V})$ & Gain & $\sigma / \mathrm{E}(\%)$ & $\mathrm{IBF}(\%)$ \\
\hline 435 & 265 & 230 & 2057 & 10.90 & 0.470 \\
\hline 445 & 260 & 225 & 2090 & 11.30 & 0.400 \\
\hline 455 & 255 & 220 & 2126 & 11.65 & 0.330 \\
\hline 465 & 250 & 215 & 2172 & 12.25 & 0.280 \\
\hline 475 & 245 & 210 & 2223 & 12.75 & 0.240 \\
\hline 485 & 240 & 200 & 2049 & 13.90 & 0.220 \\
\hline 495 & 235 & 195 & 2102 & 14.50 & 0.210 \\
\hline \multicolumn{6}{|c|}{$\mathrm{Ne}+\mathrm{CO}_{2}+\mathrm{CH}_{4}(82-9-9)$} \\
\hline $\mathrm{V}_{\mathrm{MMG}}(\mathrm{V})$ & $\Delta \mathrm{V}_{\mathrm{GEM}}^{\mathrm{top}}(\mathrm{V})$ & $\Delta \mathrm{V}_{\mathrm{GEM}}^{\mathrm{mid}}(\mathrm{V})$ & Gain & $\sigma / \mathrm{E}(\%)$ & $\operatorname{IBF}(\%)$ \\
\hline
\end{tabular}




\begin{tabular}{|c|c|c|c|c|c|}
\hline 400 & 270 & 218 & 1985 & 9.90 & 0.580 \\
\hline 415 & 265 & 215 & 2122 & 10.60 & 0.470 \\
\hline 425 & 260 & 210 & 2160 & 10.70 & 0.390 \\
\hline 435 & 250 & 205 & 1969 & 11.45 & 0.320 \\
\hline 445 & 245 & 200 & 2004 & 11.90 & 0.290 \\
\hline 455 & 240 & 195 & 2044 & 12.40 & 0.250 \\
\hline 460 & 235 & 195 & 2055 & 13.00 & 0.225 \\
\hline \multicolumn{6}{|c|}{$\begin{array}{c}\mathrm{Ne}+\mathrm{CO}_{2}+\mathrm{CF}_{4}(82-9-9) \\
\mathrm{E}_{\text {drift }}=0.4 \mathrm{kV} / \mathrm{cm}-\mathrm{E}_{\text {transfer }}=2.5 \mathrm{kV} / \mathrm{cm}-\mathrm{E}_{\text {ind }}=0.075 \mathrm{kV} / \mathrm{cm}\end{array}$} \\
\hline $\mathrm{V}_{\mathrm{MMG}}(\mathrm{V})$ & $\Delta \mathrm{V}_{\mathrm{GEM}}^{\mathrm{top}}(\mathrm{V})$ & $\Delta \mathrm{V}_{\mathrm{GEM}}^{\mathrm{mid}}(\mathrm{V})$ & Gain & $\sigma / \mathrm{E}(\%)$ & $\operatorname{IBF}(\%)$ \\
\hline 405 & 325 & 250 & 1886 & 10.00 & 0.950 \\
\hline 415 & 320 & 245 & 1908 & 10.40 & 0.800 \\
\hline 425 & 315 & 240 & 1936 & 10.70 & 0.670 \\
\hline 435 & 310 & 235 & 1965 & 11.10 & 0.560 \\
\hline 445 & 305 & 230 & 2010 & 11.30 & 0.500 \\
\hline 455 & 300 & 225 & 1984 & 11.90 & 0.420 \\
\hline 465 & 295 & 220 & 2008 & 12.50 & 0.360 \\
\hline 475 & 290 & 215 & 2030 & 13.30 & 0.310 \\
\hline \multicolumn{6}{|c|}{$\begin{array}{l}\mathrm{Ne}+\mathrm{CO}_{2}+\mathrm{CF}_{4}(82-9-9) \\
-\mathrm{E}_{\text {transfer }}=1.5 \mathrm{kV} / \mathrm{cm}\end{array}$} \\
\hline $\mathrm{V}_{\mathrm{MMG}}(\mathrm{V})$ & $\Delta \mathrm{V}_{\mathrm{GEM}}^{\mathrm{top}}(\mathrm{V})$ & $\Delta \mathrm{V}_{\mathrm{GEM}}^{\mathrm{mid}}(\mathrm{V})$ & Gain & $\sigma / \mathrm{E}(\%)$ & $\operatorname{IBF}(\%)$ \\
\hline 410 & 281 & 222 & 2361 & 9.20 & 0.960 \\
\hline 420 & 276 & 214 & 2324 & 9.55 & 0.800 \\
\hline 430 & 271 & 206 & 2281 & 9.90 & 0.670 \\
\hline 440 & 267 & 198 & 2275 & 10.20 & 0.560 \\
\hline 450 & 257 & 198 & 2385 & 10.60 & 0.470 \\
\hline 460 & 247 & 196 & 2403 & 11.25 & 0.400 \\
\hline 470 & 237 & 196 & 2507 & 12.00 & 0.330 \\
\hline
\end{tabular}

References

[1] G. V. Buren, et al., Correcting for distortions due to ionization in the STAR TPC, NIM A566 (2006) 22. doi:10.1016/j.nima.2006.05.131.

[2] W. Blum, W. Riegler, L. Rolandi, Particle Detection with Drift Chambers, 2nd Edition, Particle Acceleration and Detection, Springer-Verlag Berlin Heidelberg, 2008. doi:10.1007/978-3-540-76684-1.

[3] S. Amendolia, et al., Ion trapping properties of a synchronously gated time projection chamber, NIM A239 (1985) 192. doi:10.1016/0168-9002(85) 90714-4.

[4] H. Wieman, Gating grid concept for ALICE TPC upgrade, private correspondence (04 2014). 


\begin{tabular}{|c|c|c|}
\hline 349 & [ & URL https://wiki.bnl.gov/eic/upload/Alice_upgrade_gating_ \\
\hline 50 & & grid_idea.pdf \\
\hline 52 & [5] & $\begin{array}{l}\text { J. Mulligan, Simulations of a multi-layer extended gating grid (03 2016). } \\
\text { URL http: //arxiv.org/abs/1603.05648 }\end{array}$ \\
\hline & [6] & $\begin{array}{l}\text { F. Sauli, L. Ropelewski, P. Everaerts, Ion feedback suppression in time } \\
\text { projection chambers, NIM A560 (2006) 269. doi:10.1016/j.nima.2005. } \\
\text { 12.239. }\end{array}$ \\
\hline & {$[7]$} & $\begin{array}{l}\text { P. Colas, I. Giomataris, V. Lepeltier, Ion backflow in the micromegas TPC } \\
\text { for the future linear collider, NIM A535 (2004) } 226 \text {. doi:10.1016/j.nima. }\end{array}$ \\
\hline
\end{tabular}

[8] A. Bondar, A. Buzulutskov, L. Shekhtman, A. Vasiljev, Study of ion feedback in multi-GEM structures, NIM A496 (2003) 325. doi:10.1016/ S0168-9002(02) 01763-1.

[9] ALICE Collaboration, Technical design report for the upgrade of the ALICE time projection chamber, ALICE-TDR-016. URL https://cds.cern.ch/record/1622286

[10] ALICE Collaboration, Addendum to the technical design report for the upgrade of the ALICE time projection chamber, CERN-LHCC-2015-002. URL http://cds . cern. ch/record/1984329

[11] F. Sauli, GEM: A new concept for electron amplification in gas detectors, NIM A386 (1997) 531. doi:10.1016/S0168-9002(96)01172-2.

[12] Y. Giomataris, P. Rebourgeard, J. Robert, G. Charpak, MICROMEGAS: a high-granularity position-sensitive gaseous detector for high particleflux environments, NIM A376 (1996) 29. doi:10.1016/0168-9002(96) 00175-1.

[13] D. Neyret, et al., New pixelized micromegas detector with low discharge rate for the COMPASS experiment, Journal of Instrumentation 7 (03) (2012) C03006. doi:10.1088/1748-0221/7/03/C03006

[14] M. Vandenbroucke, Discharge reduction technologies for micromegas detectors in high hadron flux environments, Journal of Instrumentation 7 (05) (2012) C05014.

URL http://iopscience.iop.org/article/10.1088/1748-0221/7/05/ C05014

[15] S. Procureur, et al., Discharge studies in micromegas detectors in a 150 gev/c pion beam, NIM A 659 (2011) 91.

URL http://wWW.sciencedirect.com/science/article/pii/
S0168900211016548


[16] W. Anderson, et al., Design, construction, operation and performance of a hadron blind detector for the PHENIX experiment, NIM A646 (1) (2011) 35. doi:10.1016/j.nima.2011.04.015.

[17] C. Lippmann, et al., A continuous read-out tpc for the alice upgrade, presentation at Pisa 2015 meeting, Proceedings to be published in NIM (2015).

[18] A. Bay, et al., Study of sparking in micromegas chambers, NIM A488 (2002) 162. doi:10.1016/S0168-9002(02)00510-7.

[19] T. Alexopoulos, et al., A spark-resistant bulk-micromegas chamber for high-rate applications, NIM A640 (2011) 110. doi:10.1016/j.nima.2011. 03.025

[20] J. Bortfeld, Development of micromegas detectors with novel floating strip anode (04 2013).

\begin{tabular}{l} 
URL https://indico.cern.ch/event/245535/session/4/ \\
contribution/5/attachments/420744/584246/rd51MW_jbortfeldt. \\
\hline pdf
\end{tabular}

